\title{
Casein kinase 1 (CK1) promotes the proliferation and metastasis of glioma cells via the phosphatidylinositol 3 kinase-matrix metalloproteinase 2 (AKT-MMP2) pathway
}

\author{
Hua-Song Gao ${ }^{1 \#}$, She-Yu Lin ${ }^{2 \#}$, Xi Han ${ }^{1}$, Hong-Zhi Xu' ${ }^{1}$, Yi-Lu Gao ${ }^{3}$, Zhi-Yong Qin ${ }^{1}$ \\ ${ }^{1}$ Department of Neurosurgery, Huashan Hospital, Shanghai Medical College, Fudan University, Shanghai, China; ${ }^{2}$ Department of Biological \\ Sciences, School of Life Sciences, Nantong University, Nantong, China; ${ }^{3}$ Department of Neurosurgery, Affiliated Hospital of Nantong University, \\ Nantong, China \\ Contributions: (I) Conception and design: ZY Qin; (II) Administrative support: YL Gao; (III) Provision of study materials or patients: HS Gao, SY \\ Lin; (IV) Collection and assembly of data: X Han; (V) Data analysis and interpretation: HS Gao, HZ Xu; (VI) Manuscript writing: All authors; \\ (VII) Final approval of manuscript: All authors. \\ \#These authors contributed equally to this work and are co-first authors. \\ Correspondence to: Dr. Zhiyong Qin. Department of Neurosurgery, Huashan Hospital, Fudan University, Urumqi Middle Road 12\#, Shanghai 200040, \\ China. Email: zhiyongqin64@126.com.
}

Background: Glioma is a type of tumor that usually occurs in the adult central nervous system. Protein kinases have become important targets for oncotherapy since they are closely correlated with signal transduction. The role of the casein kinase 1 (CK1) gene in glioma remains to be fully elucidated.

Methods: The mRNA and protein expression of CK1 were analyzed by Realtime PCR, Western blot and immunohistochemistry. The cell behavior was assayed by MTT, Transwell and cell scratch methods. Cell cycle and cell apoptosis were performed by flow cytometer. Construction of stable cell line was completed by lentivirus infection. The nude mouse model was used for in vivo analysis on the role of CK1 by injecting the cells into subcutaneous tissue, tail vein and cerebral cortex. The prognostic role of CK1 in glioma was evaluated using Kaplan-Meier and Cox regression analyses.

Results: immunohistochemical staining demonstrated that the expression of CK1 in glioma samples was correlated with the grade of glioma. Survival analysis using Kaplan-Meier and multivariate analysis by Cox regression indicated that CK1 could be used as an independent prognostic marker for glioma. The methyl thiazolyl tetrazolium (MTT), transwell, and cell scratch assays demonstrated that the CK1 gene promoted cell proliferation and invasion through the phosphatidylinositol 3 kinase/matrix metalloproteinase 2 (AKT-MMP2) signaling pathway. In vivo experiments in mice also confirmed the ability of CK1 to enhance tumor proliferation and metastasis, with the metastatic site being the small intestine.

Conclusions: the expression of CK1 was correlated with glioma grade and patient survival and it may enhance glioma proliferation and metastasis via AKT-MMP2 pathway.

Keywords: Casein kinase 1 (CK1); glioma; proliferation; invasion; phosphatidylinositol 3 kinase/matrix metalloproteinase 2 pathway (AKT-MMP2 pathway)

Submitted Dec 30, 2020. Accepted for publication Apr 15, 2021.

doi: $10.21037 / \mathrm{atm}-21-935$

View this article at: http://dx.doi.org/10.21037/atm-21-935

\section{Introduction}

Glioma is a type of tumor located in the adult primary central nervous system. The median survival for patients with glioma is approximately 2 years. Despite abundant research and great advances in radiation therapy and chemotherapy, the prognosis for patients remains poor (1). Current treatments 
for glioma are hindered by many obstacles, including tumor hypoxia which results in therapeutic resistance. Furthermore, the invasiveness of gliomas into normal brain tissues often results in incomplete tumor removal (2). Therefore, improved understanding of the features and molecular mechanisms involved in glioma development and progression, as well as the discovery of novel molecular sites is crucial to facilitate the development of better therapies for patients with glioma.

Since protein kinases are heavily involved in signal transduction (3), they have become important therapeutic targets in the treatment of cancers. Through reversible phosphorylation of their target proteins, kinases can change their activity, function, and localization and thus are integrally involved in various cellular processes. The casein kinases (CK) are a group of serine/threonine kinases that function as a regulator in the signal transduction of eukaryotic cells (4). In recent years, increasing evidence have shown that CK1 is involved in cancer progression across different types of tumors (5) including breast cancer, kidney cancer, and colorectal cancer (6). However, the precise role of CK1 and whether CK1 can be an effective target for the therapy of glioma remains elusive.

The phosphatidylinositol 3 kinase (PI3K)/AKT pathway is activated in more pathway components and in more tumor types than in any other signaling pathway involved in cancer (7). AKT belongs to the serine/threonine group of kinases and has three isoforms. Among them, AKT1 and AKT2 are widely express, whereas the expression of AKT3 is restricted to the brain and testes. Activation of phosphorylated AKT (p-AKT) is involved in many biological processes including cell proliferation, cell growth, anti-apoptosis, and cell cycle progression (8). AKT can be activated by a wide variety of tyrosine kinases such as CK1, cytokine receptors, and G-protein coupled receptors (9).

The matrix metalloproteinases (MMPs) play an important role in the degradation of the extracellular matrix (ECM), which is involved in many cancer-related processes including cell migration, invasion, metastasis, and proliferation (10). The MMP family is a large class of multi-domain, zinc-dependent endopeptidases, and includes at least 28 members (11). Among them, MMP2 has been shown to be associated with the invasion and metastasis of many tumors (12).

This study identified CK1 as an independent prognostic marker for glioma and demonstrated that CK1 promotes glioma proliferation and metastasis in an AKT/phosphorylated-
AKT (p-AKT)/MMP2 signaling pathway-dependent manner.

We present the following article in accordance with the ARRIVE reporting checklist (available at http://dx.doi. org/10.21037/atm-21-935).

\section{Methods}

\section{Reagents}

Reagents for cell behavior analysis such as dimethyl sulfoxide (DMSO), trypsin, propidium iodide, and methyl thiazolyl tetrazolium (MTT) were purchased from Beyotime (Haimen, China). The transwell invasion chamber was obtained from Corning Corp. (Shanghai, China). The kits for protein extraction and quantification were from Beyotime (Haimen, China). The rabbit polyclonal antibodies for CK1, glyceraldehyde 3-phosphate dehydrogenase (GAPDH), and MMP2 were purchased from Abcam (Cambridge, UK), Boshide (Wuhan, China), and Lifespan Biosciences (Seattle, USA), respectively. The secondary horseradish peroxidase (HRP) conjugated antibodies, rabbit anti-mouse and goat anti-rabbit antibodies, were purchased from Life Technologies (CA, USA). The ECL chemiluminescence kit was obtained from Boshide (Wuhan, China). All cell culture components were purchased from Corning Corp. (Shanghai, China).

\section{Patients and follow-up}

Glioma tissues $(\mathrm{n}=180)$ were collected from patients who were admitted to the Affiliated Hospital of Nantong University with newly diagnosed astrocytomas between 2010 and 2016. These patients did not receive therapy prior to sample collection. Normal brain tissues were acquired from 15 patients with epilepsy who underwent surgery. These samples were reviewed to verify the absence of tumors. After resection, all specimens were gathered in the operating room and immediately stored at $-80{ }^{\circ} \mathrm{C}$. In this study, the enrollment criteria for patients were as follows: pathology diagnosis of glioma based on WHO grading; and no prior anti-glioma treatment. This study was approved by the research ethics committee of the Affiliated Hospital of Nantong University and written informed consent was obtained from each patient. All procedures were conducted in accordance with the Declaration of Helsinki (as revised in 2013). Patients were followed-up every 2 months during the first post-operative year and thereafter at least every 
3-4 months until September 15, 2017. The median value of follow-up was 27 months (range from 3 to 64 months). Overall survival (OS) was defined as the length of time from surgery to death or the time the last observation was taken. Data regarding surviving patients were censored at the final follow-up. The patients were divided into two groups based on high or low CK1 expression as assessed via immunohistochemical staining.

\section{Immunobistochemistry}

Samples were dissected, fixed in $4 \%$ paraformaldehyde for 12 hours at $4{ }^{\circ} \mathrm{C}$, and dehydrated in $20 \%$ sucrose (12 hours at $4{ }^{\circ} \mathrm{C}$ ) and $30 \%$ sucrose $\left(72\right.$ hours at $\left.4{ }^{\circ} \mathrm{C}\right)$. Afterwards, tissues were sectioned on a cryostat set at $10 \mu \mathrm{m}$ thickness. Endogenous peroxidase was inactivated by treatment with $3 \%$ hydrogen peroxide followed by epitope retrieval with heat in ethylenediaminetetraacetic acid (EDTA) buffer for 15 minutes. The slides were blocked in phosphate buffered saline (PBS) containing $5 \%$ bovine serum albumin (BSA) and $0.1 \%$ Triton $\mathrm{X}-100$ for 1 hour at room temperature, followed by incubation with primary antibodies at $4{ }^{\circ} \mathrm{C}$ for 12 hours. The slides were then incubated with a biotinconjugated secondary antibody and reacted with the avidinperoxidase complex for 1 hour. Diaminobenzidine (DAB) was applied as the chromogenic agent and the nuclei were counterstained with hematoxylin. Lastly, the slides were covered with a coverslip and mounting medium. The following primary antibodies were used: rabbit anti-CK1, rabbit anti-AKT, and anti-p-AKT (Suzhou, USA).

\section{Cells culture}

The glioma cell lines U251, U87MG, and U373 were obtained from the cell bank of the Chinese Academy of Sciences. Cells were cultured in Dulbecco's modified eagle's medium (DMEM) supplemented with $10 \%$ fetal bovine serum (FBS), $2 \mathrm{mM}$ glutamine, $50 \mathrm{U} / \mathrm{mL}$ penicillin, and $50 \mathrm{U} / \mathrm{mL}$ streptomycin, in a humidified atmosphere under $5 \% \mathrm{CO}_{2}$ at $37{ }^{\circ} \mathrm{C}$. The stable cell lines were established by lentivirus infection and divided into three groups including the overexpression group (CK1 overexpression), the interference group [CK1 knockdown by short hairpin RNA (shRNA)] and the control group (empty vector lentivirus infection). The AKT inhibition experiments were performed by adding the AKT inhibitor API-1 $(10 \mu \mathrm{M})$ to the above overexpression groups. After culture for 48 hours, western blot analysis, MTT assay, and transwell experiments were performed.

\section{Western blot analysis}

Protein expression in the glioma samples and cultured cells was examined by Western blot analysis. The total protein was obtained using Tissue Protein Extraction Reagent (Pierce Chemical Co., USA) and equal amounts were separated on a $10 \%$ sodium dodecyl sulfate-polyacrylamide gel. The proteins were then transferred onto nitrocellulose membranes (Waterman, USA) using a Mini Trans-Blot Apparatus (BioRad, USA), followed by blocking with Tween-PBS (TPBS) containing $5 \%$ skim milk powder for 3 hours. Subsequently, the membranes were incubated with anti-CK1 antibodies or antiGAPDH antibodies overnight at $4{ }^{\circ} \mathrm{C}$. The membranes were then washed 3 times with PBS containing $0.05 \%$ Tween-20 and incubated with secondary antibodies conjugated with HRP for 1 hour. Protein levels were detected by incubating the membranes with ECL reagents according to the manufacturer's specifications (Boshide, Wuhan city, China).

\section{Apoptosis analysis}

The apoptosis of glioma cells was assessed by flow cytometry. Briefly, $3 \times 10^{6}$ cells were collected by trypsin digestion and centrifuged at 1,400 rpm. The pellet was then resuspended in PBS and stained with $15 \mu \mathrm{L} \mathrm{PE}$ (phycoerythrin) and $15 \mu \mathrm{L}$ 7-AAD (7-amino actinomycin D). Subsequently, the samples were analyzed using the flow cytometer (BD Biosciences). The percentage of apoptotic cells was determined. Intact cells stain $\mathrm{PE}^{-} / 7-\mathrm{AAD}^{-}$, early apoptotic cells stain $\mathrm{PE}^{+} / 7-\mathrm{AAD}^{-}$, and late apoptotic or necrotic cells stain $\mathrm{PE}^{+} / 7-\mathrm{AAD}^{+}$. Results were analyzed by the Software Muse 1.5. The experiment was performed in triplicates.

\section{MTT assay}

Cells were seeded into 96 -well plates at the density of $3 \times 10^{4}$ cells $/ \mathrm{mL}$, in a total volume of $100 \mu \mathrm{L}$ for each well, and allowed to adhere overnight. 48 hours after treatment, $100 \mu \mathrm{L}$ MTT $(0.5 \mathrm{mg} / \mathrm{mL})$ was added to each well and the cells were cultured for another 4 hours. The medium was then removed and $100 \mu \mathrm{L}$ DMSO was added to dissolve the MTT-formazan crystals. Optical density (OD) values were measured at a wavelength of $570 \mathrm{~nm}$ with a microplate reader. The experiment was repeated three times.

\section{Cell cycle analysis}

Cells were seeded in six-well plates at the density of 
$3 \times 10^{5}$ cells $/ \mathrm{mL}$, in a total volume of $2 \mathrm{~mL}$ for each well. 48 hours after treatment, the cells were washed with PBS three times. Cells were then detached by trypsinization, followed by two PBS washes and fixed in $70 \%$ cold ethanol overnight at $-20{ }^{\circ} \mathrm{C}$. After washing with citrate phosphate buffer and PBS, the cells were treated with RNase solution $(100 \mu \mathrm{g} / \mathrm{mL})$ for 30 minutes at $37^{\circ} \mathrm{C}$. Propidium iodide was then added for 30 minutes at room temperature to dye the cells. The cell cycle was analyzed by flow cytometry (BD Biosciences, CA, USA) and the results were analyzed by the software mflt32.

\section{Cell invasion and scratch assay}

The transwell experiments were performed to assess the invasive ability of cells by examining the number of cells passing through a polycarbonate membrane $(8 \mu \mathrm{m}$ pore size). Briefly, after washing with serum-free medium, the chamber was covered with $20 \mu \mathrm{L}$ Matrigel to create the Matrigel membrane. The chamber was divided into an upper and a lower room. The cells were seeded in the upper room and cultured for 24 hours. The cells on the upper surface of membrane were then removed using a cotton swab and the cells invading through the membrane were stained with $1 \%$ crystal violet. The cells that migrated through the polycarbonate membrane were counted under a Leica microscope in 8 random fields of view.

For the scratch assay, six-well plates were seeded with cells and a "wound" line was created using a sterile $200 \mu \mathrm{L}$ pipette tip. The width of the wound was detected under a microscope at different time points to assess the migration ability of the cells. The Student $t$-test was used for statistical analysis.

\section{Animal model}

Athymic BALB/c nude mice were purchased from the Shanghai Institute of Material Medicine and housed in specific pathogen-free conditions with sterile food and water. Animal care and experimental protocols were conducted following the guidelines established by the Shanghai Medical Experimental Animal Care Commission and study involving animals approved by the Ethics Committee of Huashan Hospital affiliated to Fudan University (No. 2016-111). The xenografts models were performed by injecting the stable tumor cell lines subcutaneously or into the cerebral cortex. Mice were randomly divided into different groups, with 6 mice in the subcutaneous injection group, 5 mice in cerebral cortex injection, and 5 mice in the tail vein injection group. The three kinds of xenografts models all include three groups.

The subcutaneous injections were performed by pinching the nape of the mouse using the index finger and thumb. The cells were then injected into the pouch created by pulling the skin away from the body. The xenografts for the cerebral cortex were performed with the stereotactic method. Briefly, 6-week-old nude mice were anesthetized with sterile saline containing $100 \mathrm{mg} / \mathrm{kg}$ ketamine and $5 \mathrm{mg} / \mathrm{kg}$ xylazine. The intracranial injection was performed using a Hamilton syringe with a 33-gauge needle (Hamilton, Reno, NV) that was controlled by a Micro 4 Microsyringe Pump Controller (World Precision Instruments, Sarasota, FL). The injection speed was $0.4 \mu \mathrm{L} / \mathrm{min}$. The glioma cells were injected into the mid-left striatum at the following coordinates in $\mathrm{mm}$ from bregma: +0.5 anterior-posterior, +2.5 medio-lateral, and -2.5 dorso-ventral. After 6 weeks, all three groups formed tumors. The weight and size of the brain tumors were measured. The metastasis model was carried out by tail vein injection of the tumor cells. After 8 weeks, all mice were euthanized and the tumor metastasis was imaged using the live image system. In addition, tissue samples were excised for hematoxylin and eosin (HE) staining.

\section{Statistical analysis}

The SPSS 17.0 software (SPSS) was applied for statistical analyses. The Pearson $\chi^{2}$ test was selected to evaluate the correlation between CK1 expression and the clinical pathological characteristics. The recurrence interval was measured from the glioma resection to the first radiological evidence of recurrence. All deceased patients were censored to confirm whether patients experienced recurrence if they were in the absence of recurrence diagnose. The cumulative recurrence and survival rates were analyzed by the KaplanMeier method with the log-rank test. The Cox proportional hazards regression model was applied to univariate and multivariate analyses. Other data were assayed by $t$-test or one-way analysis of variance (ANOVA). All P values were two-sided and the results were considered statistically significant when $\mathrm{P}<0.05$.

\section{Results}

\section{The expression of CK1 in glioma patient specimens and different cell lines, and its correlation with prognosis and cell behavior}

To investigate whether CK1 is important in determining 
Table 1 Correlation between CK1 staining and clinicopathologic characteristics in 180 glioma patients

\begin{tabular}{lccc}
\hline \multirow{2}{*}{ Variables } & \multicolumn{2}{c}{ CK1 staining, N (\%) } & P \\
\cline { 2 - 3 } & High, $103(57.2 \%)$ & Low, 77 (42.8\%) & \\
\hline Age & & & 0.529 \\
$>50$ & 38 & 24 & \\
Gender & 65 & 53 & 0.652 \\
Male & & & \\
Female & 55 & 44 & \\
WHO grade & 48 & 33 & 0.006 \\
I/II & & & \\
III/IV & 15 & 25 & \\
Ki-67 staining & 88 & 52 & \\
High & 63 & 35 & \\
Low & 40 & 42 & \\
\hline
\end{tabular}

the clinical outcomes of glioma patients, its expression was assessed in 180 glioma patients. The Pearson $c^{2}$ test indicated that CK1 expression was strongly associated with clinicopathologic characteristics including Ki-67 levels and $\mathrm{WHO}$ grade $(\mathrm{P}<0.05$; Table 1$)$. Immunohistochemical studies also clearly demonstrated that CK1 expression in patient specimens was upregulated in samples with increased tumor grade, suggesting that CK1 expression is closely related to tumor grade (Figure 1A). However, other clinicopathologic characteristics such as age and gender, were not correlated with the expression of CK1.

In this study, the last follow-up was in September 2017. At this time, $80.5 \%$ (145 of 180) of patients had suffered recurrence and $58.8 \%$ (106 of 180) had died with recurrence. The 1 - and 3-year overall survival (OS) rates in patients with high CK1 expression were significantly lower than those in patients with low CK1 expression ( $87.0 \%$ vs. $92.7 \%$, and $37.4 \%$ vs. $51.4 \%$, respectively). In addition, the 1 - and 3 -year cumulative recurrence rates were also markedly higher in the patients with high CK1 expression compared to patients with low CK1 expression (32.4\% vs. $16.8 \%$ and $65.7 \%$ vs. $47.8 \%$, respectively; Figure 1B).

To confirm the relationship between the expression of CK1 and cell behavior, the different glioma cell lines were cultured and the protein levels of CK1 were detected by western blot analysis (Figure $1 C$ ). Cell viability and migration ability were also analyzed. The levels of CK1 protein expression in the U87MG cell line and the U373 cell line were higher than that in the U251 cell line. The U87MG cell line also showed better cell viability compared to the other two cell lines (Figure 1D,E). Moreover, the U87MG cell line demonstrated better migration ability compared to the other two cell lines in the scratch experiments. Together, these results suggested that the CK1 gene plays an important role in cell behavior and may influence patient prognosis.

\section{The CK1 gene promotes the proliferation and migration of glioma cells}

To investigate the role of CK1 in glioma, overexpression of the CK1 gene in U87MG cell line was achieved by lentivirus infection. In the interference group, CK1 was knocked down using short hairpin RNAs. The empty lentivirus vector was used as a control. After infection, the levels of CK1 protein in the different stable cell lines (overexpression group, interference group and control group) were examined by western blot analysis. Protein levels of CK1 in the overexpression group were markedly higher than the interference group and the control group. CK1 protein expression in the interference group was significantly inhibited compared to the other two groups (Figure 2A). These results demonstrated that the stable cell lines were successfully established.

The effects of CK1 overexpression were determined via the MTT assay, the cell cycle assay, the cell apoptosis assay, the transwell experiments, and the scratch tests. Overexpression of the CK1 gene markedly improved the proliferation of glioma cells compared to the other two groups (Figure 2B). Furthermore, based on the cell cycle analysis, the proliferation index (PI) was significantly greater in cells overexpressing CK1 compared to control cells and cells in which CK1 was silenced (Figure 2C). In addition, the percentage of apoptotic cells in the CK1 overexpression group was significantly lower than that in the control group and the interference group (Figure 2C,D). These results suggested that the CK1 gene increased cell proliferation and inhibited cell apoptosis.

To ascertain the relationship between CK1 and cell migration, the scratch and invasion experiments were conducted. Inhibition of CK1 expression in U87MG cells reduced the motility of glioma cells while overexpression of CK1 enhanced glioma cell motility (Figure 2E,F). 
A

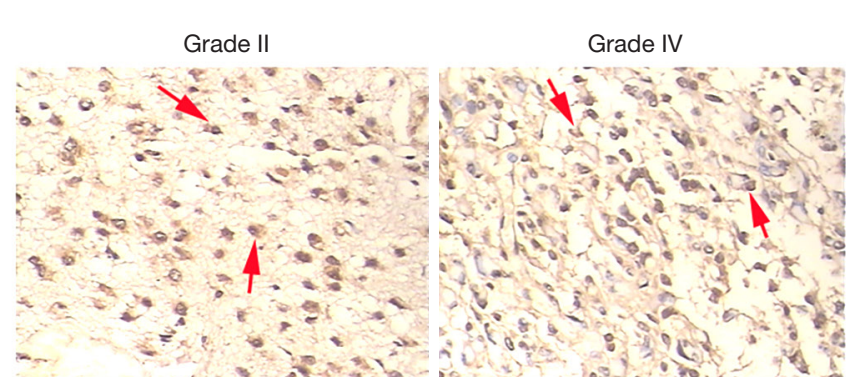

B

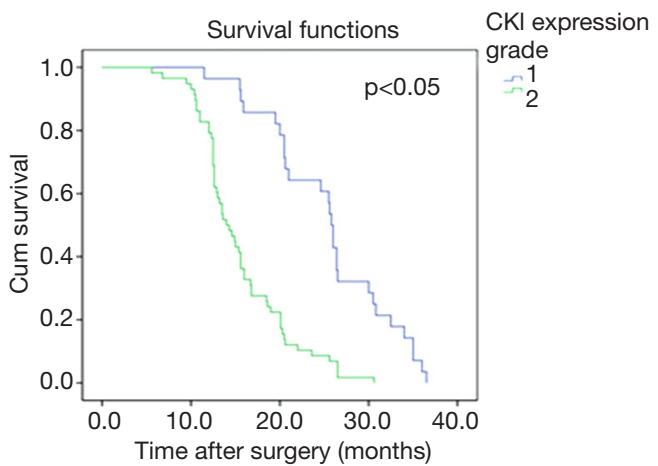

C
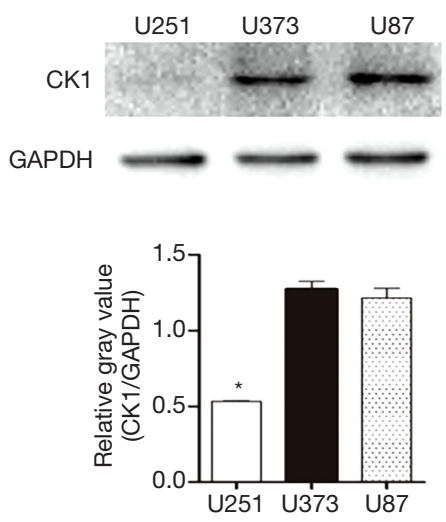

D

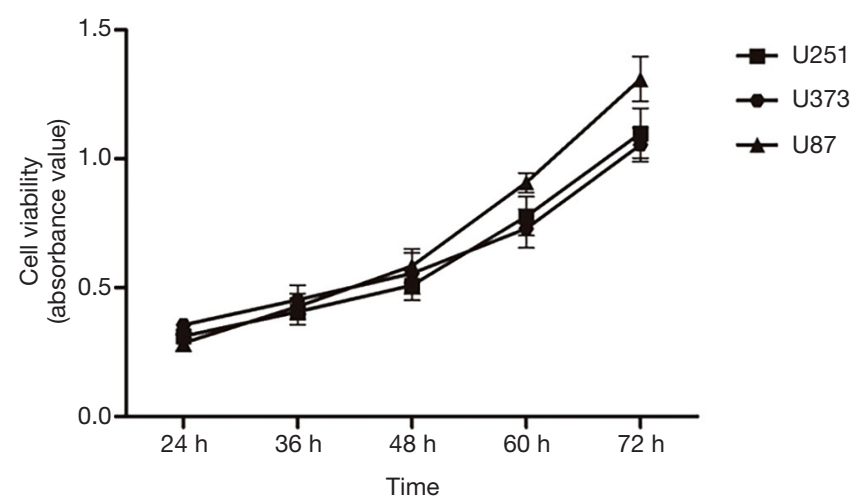

$\mathrm{E}$

U251
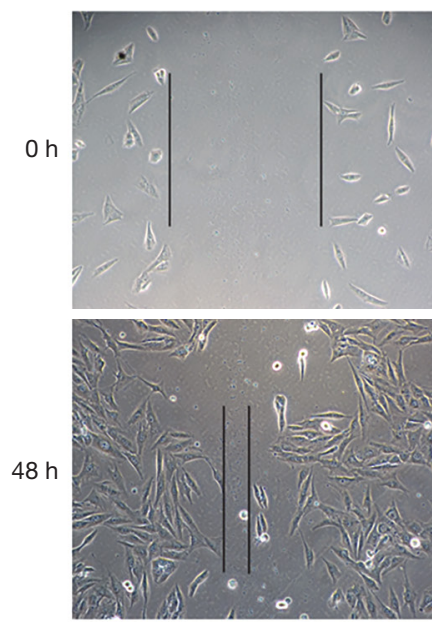

U373
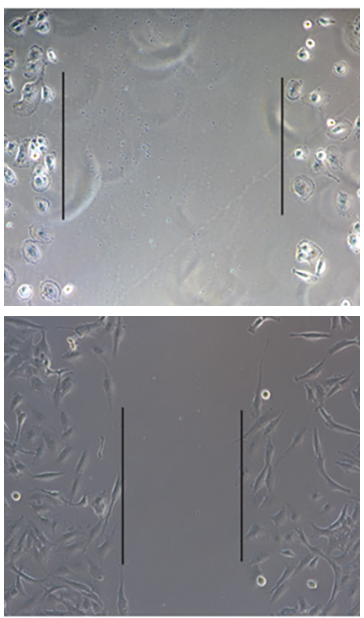

U87
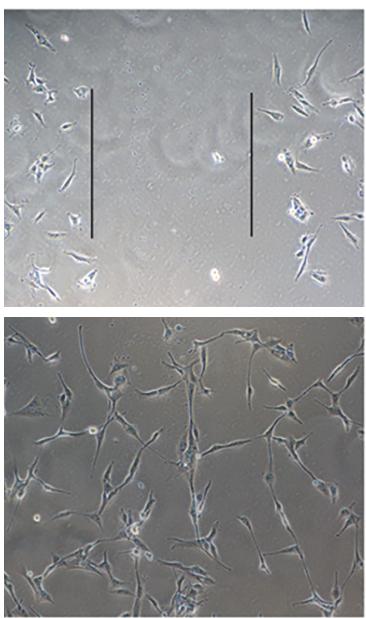

Figure 1 Upregulation of CK1 was associated with poor prognosis and was involved in glioma cell behavior. (A) Representative images of CK1 staining by immunohistochemistry in tumor tissues from patients diagnosed with grade II and grade IV glioma (200x). The red arrow indicates a significant difference $(\mathrm{P}<0.05)$. (B) Kaplan-Meier survival curve for glioma patients stratified by low and high CK1 expression. (C) Representative western blots showing the expression levels of CK1 in U251, U373, and U87MG glioma cell lines. The gray value statistics graph is shown below. * indicates a significant difference $(\mathrm{P}<0.05)$. (D) Cell viability of glioma cell lines analyzed at $24,36,48$, and 60 hours. (E) The wound closure ability of cell lines was analyzed in scratch experiments (200×). 
A

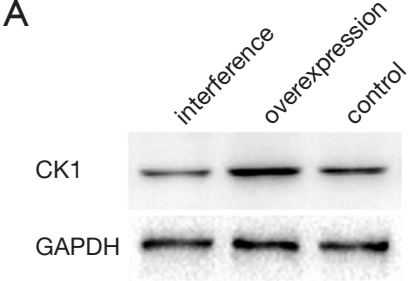

B

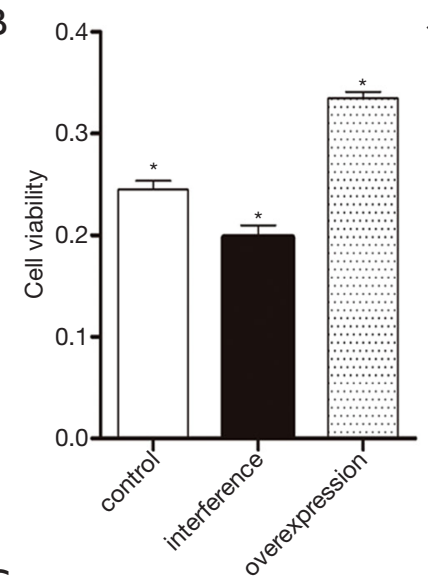

C
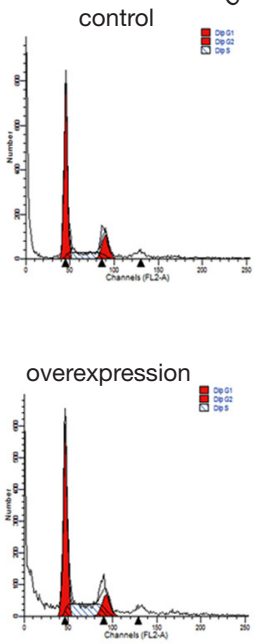
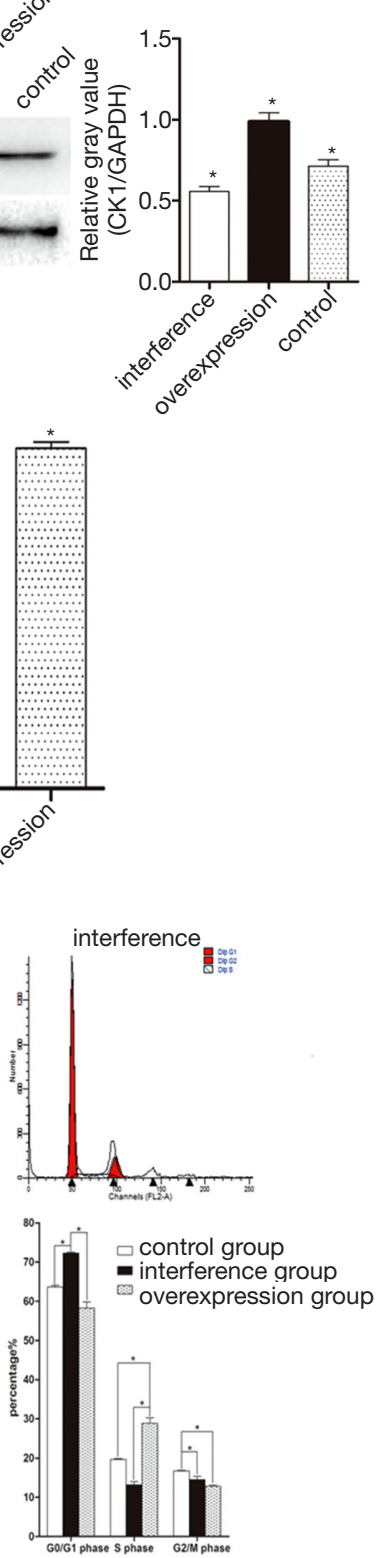

D

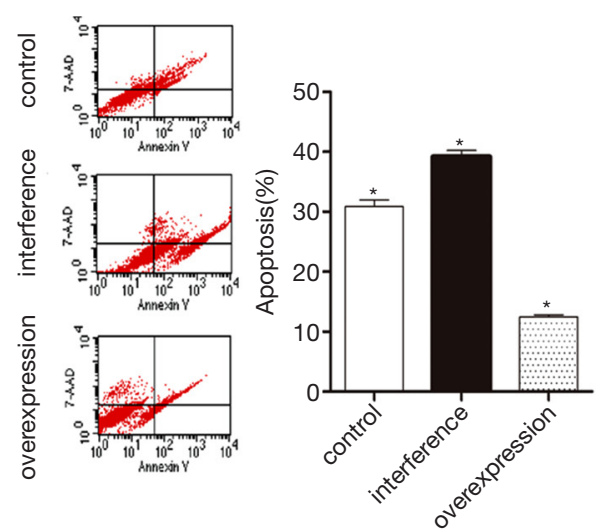

$\mathrm{E}$
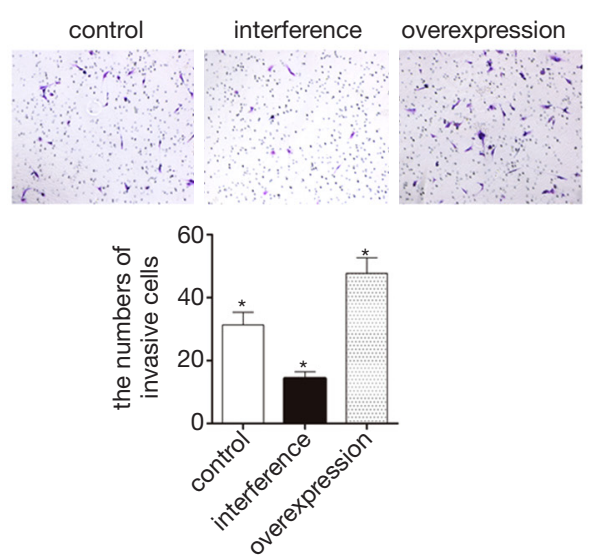

$\mathrm{F}$

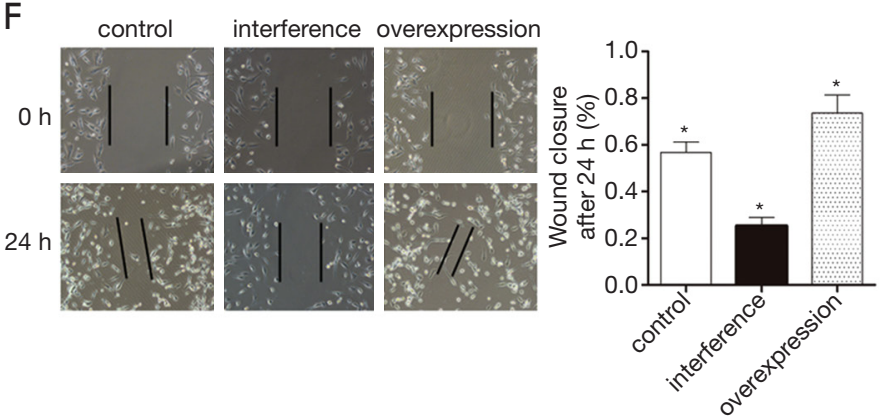

Figure 2 Augmenting CK1 expression promotes the proliferation and migration of glioma. (A) Immunoblot of CK1 in stable cell lines including control, overexpression, and knockdown groups. The gray value statistics graph is shown on the right, ${ }^{*}$ indicates a significant difference $(\mathrm{P}<0.05)$. (B) The cell viability assay was carried out by the MTT method on the indicated groups. * indicates a significant difference $(\mathrm{P}<0.05)$. (C) The cell cycle analyzed by flow cytometry showed that the percentage of cells in the G1/G0 phase in the CK1 overexpression group was significantly lower than that in the other two groups. The results suggest that the proliferation index (PI) in the CK1 overexpression group was higher than that in other two groups. * indicates a significant difference $(\mathrm{P}<0.05)$. (D) Flow cytometry analysis showed that the percentage of apoptotic cells in the CK1 overexpression group was lower than that in the other two groups. * indicates a significant difference $(\mathrm{P}<0.05)$. $(\mathrm{E})$ The invasion assay indicated that the invasion ability in the interference group was lower than that in the other two groups (200x). Cells were stained with crystal violet solution. * indicates a significant difference $(\mathrm{P}<0.05)$. (F) Scratch assay revealed that the downregulation of CK1 leads to a significant decrease in the migratory ability of U87 cells (200x). ${ }^{*}$ indicates a significant difference $(\mathrm{P}<0.05)$. 
A

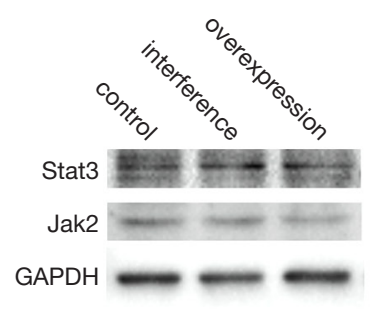

B

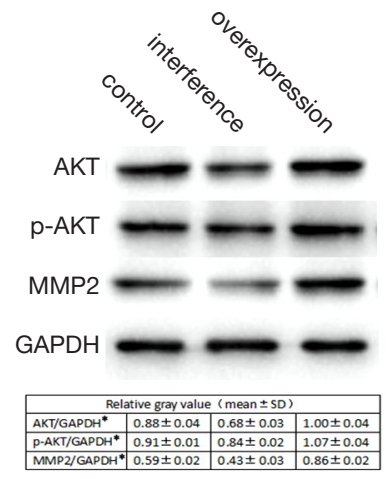

C

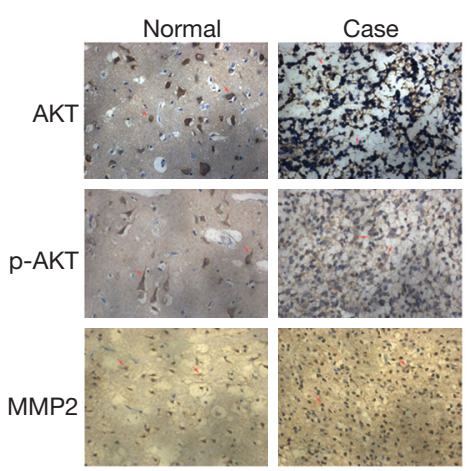

Figure 3 The CK1-mediated cell proliferation and metastasis closely correlates with the expression of AKT, p-AKT (Thr-308), and MMP2. (A) Immunoblot analysis demonstrated that the levels of STAT3and JAK2 protein expression were not affected by CK1 expression. (B) Western blot analysis demonstrated that the protein expression of AKT, p-AKT, and MMP2 were significantly affected by variations in CK1 expression. The gray value statistics table is shown below. * indicates a significant difference $(\mathrm{P}<0.05)$. (C) Representative images of AKT, $\mathrm{p}-\mathrm{AKT}$, and MMP2 staining by immunochemistry in tissues from patients $(200 \mathrm{x})$. The red arrow indicates a significant difference $(\mathrm{P}<0.05)$.

\section{The AKT pathway and MMP2 are involved in the proliferation and migration promoted by CK1}

The role of the STAT3/Jak2 and AKT pathways in the CK1-mediated effects was investigated. No significant differences in the STAT3/Jak2 pathway were observed among the three stable cell lines (Figure $3 A$ ). However, a significant difference was detected in the expression of AKT and its phosphorylated products among the three groups (Figure 3B).

MMP2 is an important tumor-related factor and has been shown to participate in tumor metastasis by degrading the ECM (13). Therefore, the protein levels of MMP2 were measured by western blot analysis. Cells overexpressing CK1 demonstrated significantly higher expression of MMP2 compared to control cells and cells in which CK1 had been silenced (Figure 3B).

To further ascertain whether AKT, p-AKT, and MMP2 are involved in glioma cell proliferation and metastasis, immunohistochemistry was performed on brain specimens from patients with glioma and control patients. AKT, p-AKT, and MMP2 staining was observed in the cytoplasm of tumor cells, and their expression was significantly increased compared to the control samples (Figure 3C).

\section{The CK1-mediated cell growth and migration is facilitated by $A K T$ and its phosphorylation}

To confirm that AKT is involved in CK1-mediated cell proliferation and metastasis, the stable cell line overexpressing CK1 was treated with the AKT inhibitor API-1 (the API-1 group). Protein levels of AKT, p-AKT, and MMP2 were detected by western blot analysis. The protein levels of AKT and p-AKT were reduced by the inhibitor API-1. The MMP2 expression was also reduced following treatment with API-1 compared to untreated cells (Figure 4A). These results suggested that CK1 regulates the expression of MMP2 via the AKT pathway. Moreover, cell viability was markedly inhibited by API-1 (Figure 4B). The transwell assay demonstrated that API-1 noticeably reduced the number of cells migrating to the other side of the membrane (Figure 4C).

These results suggested that AKT and p-AKT were involved in the CK1-mediated proliferation and metastasis of glioma cells. Furthermore, CK1 regulated the expression of MMP2 through the AKT pathway, suggesting that the effects of CK1 on glioma cell proliferation and migration is dependent on both AKT and MMP2.

\section{CK1 promotes glioma growth and metastasis in vivo}

To evaluate whether CK1 influences glioma growth and metastasis in vivo, xenograft models were created by injecting the stable tumor cell lines subcutaneously or into the cerebral cortex of mice. The tumor weight in both the subcutaneous xenograft and the cerebral cortex xenograft were significantly greater in animals injected with cells overexpressing CK1 compared to animals injected with control cells or cells in which CK1 had been silenced (Figure $5 A, B$ ). In addition, the stable cell lines were injected 
A

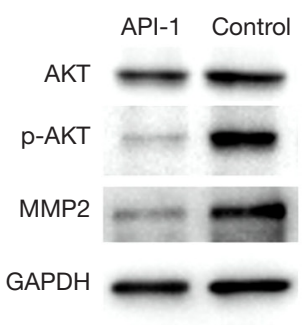

B

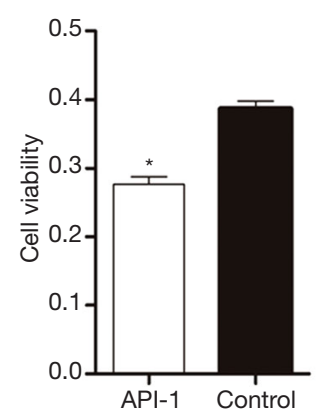

C
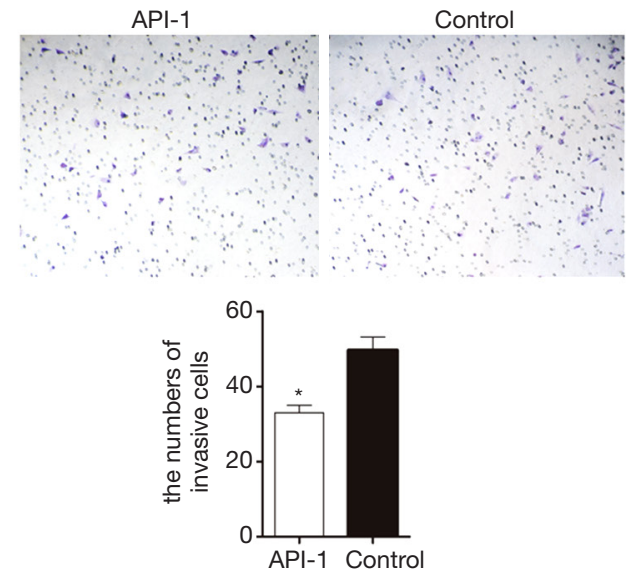

Figure 4 AKT and MMP2 were found to participate in the ability of CK1 to promote cell proliferation and metastasis. (A) The CK1 overexpression cell line was cultured in $10 \mu \mathrm{m} / \mathrm{mL}$ API-1 (API-1 group) or the equivalent amount of phosphate buffered saline (PBS) (control group) for 48 hours. Immunoblot analysis showed that the expression of AKT, p-AKT, and MMP2 was reduced in the API-1 group compared to the control group. (B) Cell viability assay showed that API-1 repressed cell proliferation. * indicates a significant difference $(\mathrm{P}<0.05)$. (C) The invasion experiment demonstrated that API-1 reduced cell invasion ability $(200 \times)$. Cells were stained with crystal violet solution. * indicates a significant difference $(\mathrm{P}<0.05)$.

into the tail veins of nude mice to generate the tumor metastasis model. The in vivo image system revealed that CK1 overexpression enhanced tumor metastasis, with the metastatic site being the small intestine. The HE staining experiments further confirmed that the metastatic tumor cells had formed a new neoplasm in the small intestine (Figure 5C).

\section{Discussion}

Gliomas are the most common primary malignant brain tumor in adults. Low-grade gliomas (WHO grades I or II) are well-differentiated and have a good prognosis. Highgrade gliomas (WHO grades III or IV) are undifferentiated and have a poor prognosis (14). However, to date, there is no complete cure for gliomas (15).

CK1 family members usually catalyze the transfer of phosphate on to Ser/Thr residues of their protein substrates. Its isoforms have been reported to be involved in numerous, key signaling pathways involved in regulating proliferation, cell growth and viability, both during development and in tissue homeostasis (16). CK1 (also named CSNK1 $\alpha 1$ ), as a serine/threonine- specific protein kinase, often functions in multiple cellular processes such as gene transcription, cell division, and membrane transport. Increasingly, CK1 has been shown to promote tumor development and therefore it has become a potential therapeutic target (17). However, the precise role of CK1 in gliomas remains elusive. This current study demonstrated that CK1 is highly expressed in a substantial proportion of gliomas and its expression is clearly associated with the grade of gliomas. Survival analysis revealed that overexpression of CK1 in gliomas was associated with a shorter OS. These findings suggested that CK1 could be used as a marker for tumor aggressiveness and a predictor for prognosis in glioma patients.

Differential expression of CK1 was detected in 3 glioma cell lines, with the U87MG and U373 cell lines expressing higher protein levels of CK1. Interestingly, the U87MG cell line also showed higher cell viability and invasion ability. Taken together, the results suggested that the CK1 gene was possibly involved in cell proliferation and invasion. This was further verified by the cell model experiments using the U87MG cell line. High CK1 expression was also detected in the U373 cell line and this corresponded to lower cell proliferation and migration ability. On the contrary, the U251 cell line showed a higher migration ability compared to the U373 cell line. This suggested that the ability of CK1 to promote glioma proliferation and invasion is influenced by many other factors and improved understanding of the detailed mechanisms is warranted. Among the isoforms, the Casein Kinase 1 Epsilon was reported to regulate glioblastoma cell survival and have the potential to be treatment target (18).

It is interesting to note that the percentage of apoptotic 
A

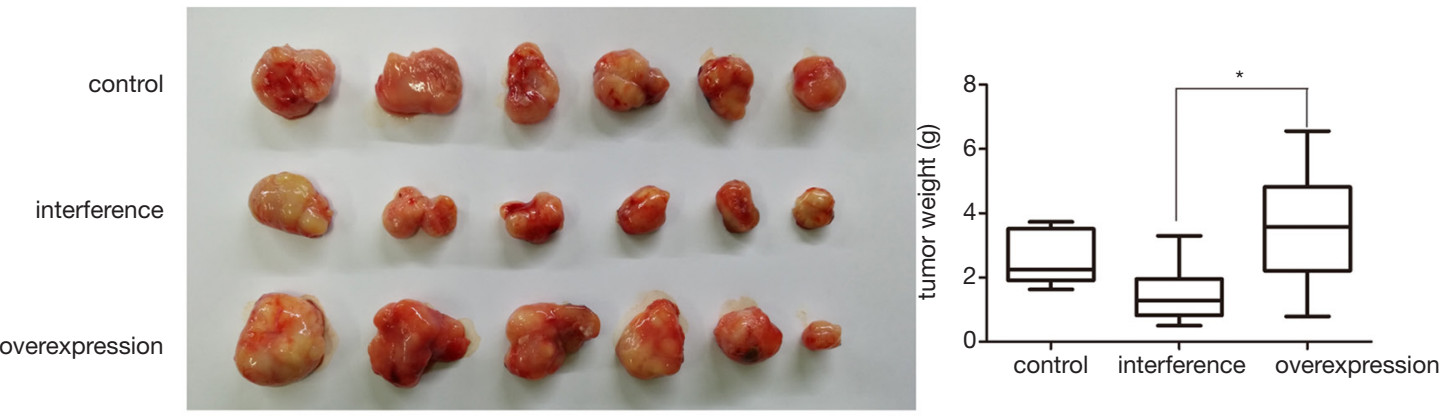

B

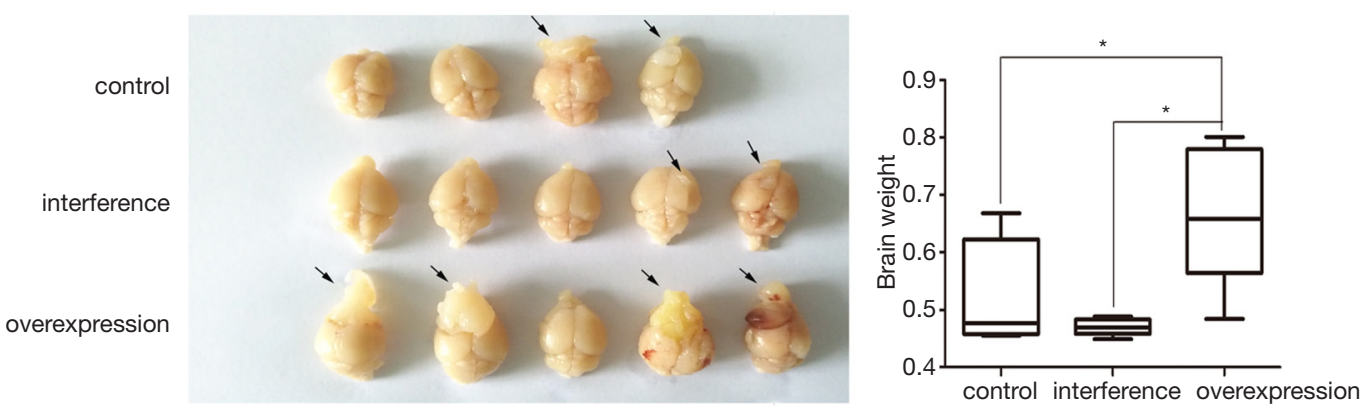

C

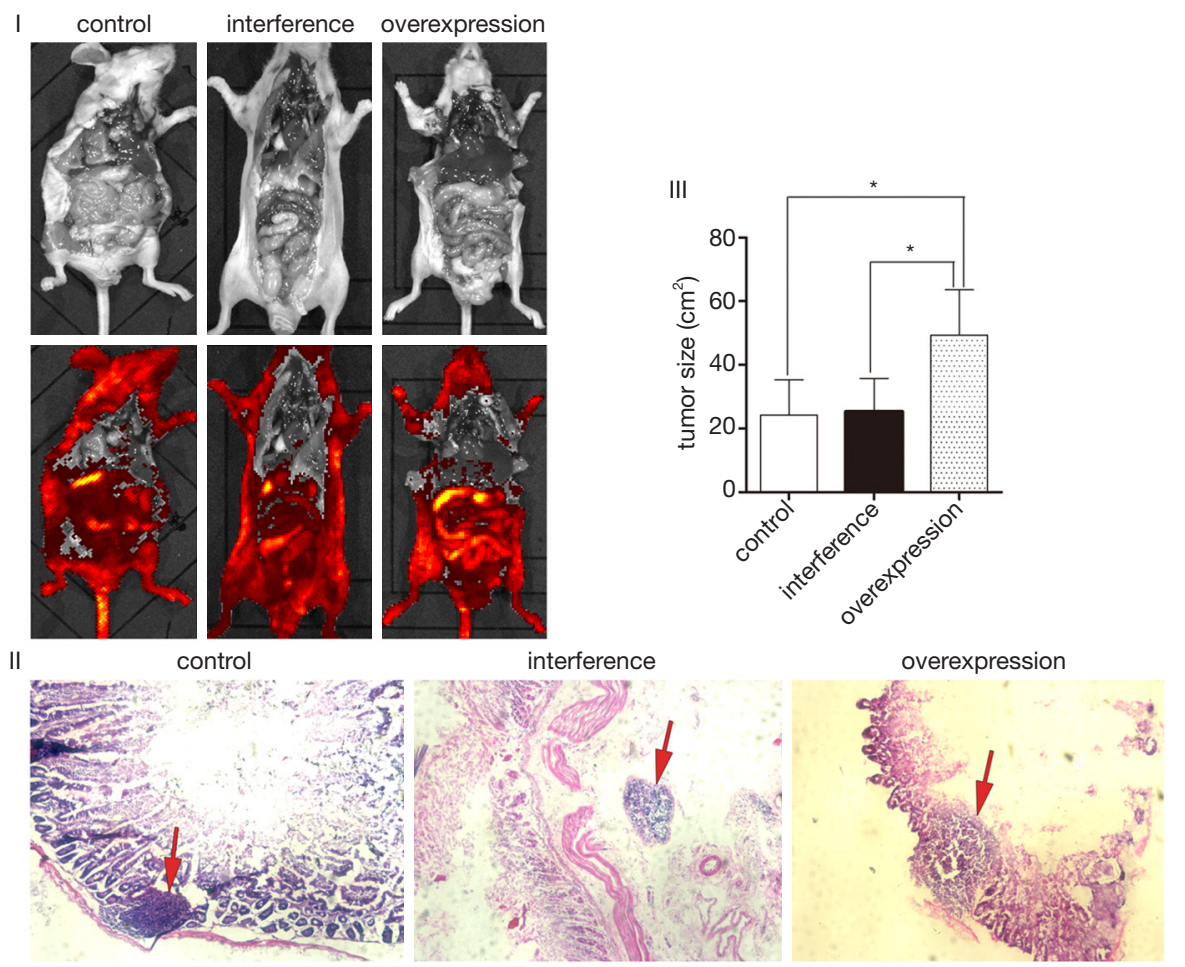

Figure $5 \mathrm{CK} 1$ promoted glioma growth and metastasis in an in vivo model using nude mice. (A) The subcutaneous xenografts model on nude mice showed that overexpression of CK1 augmented the proliferation of glioma cells. ${ }^{*}$ indicates a significant difference $(\mathrm{P}<0.05)$. $(\mathrm{B})$ The stereotactic injection of tumor cells in the cerebral cortex of mice revealed that CK1 increased the growth of glioma cells. Black arrow and * indicate a significant difference $(\mathrm{P}<0.01)$. (C) Injection of glioma cell lines into the tail veins of mice showed that CK1 promoted metastasis of the glioma to the small intestine. HE staining showed that the CK1 overexpression group had larger tumors compared to the control group and the group with suppressed CK1 expression (200x). Red arrow and * indicate a significant difference $(\mathrm{P}<0.05)$. 
cells reached $30 \%$ in the control group. The lentivirus which was used to develop the stable cell lines contains the green fluorescent protein (GFP), and this can sometimes cause the flow cytometer to consider some normal cells as apoptotic cells.

To date, many pathways were reported to be involved in the proliferation and metastasis of glioma such as Wnt/ $\beta$-catenin pathway and FAK/Akt/MMPs pathways $(19,20)$. To determine the pathways involved in the CK1-mediated glioma cell proliferation and metastasis, the STAT3/Jak2 and the AKT pathways were examined. No differences were found in the protein levels of STAT3 and Jak2 between cells overexpressing CK1, cells in which CK1 had been silenced, and control cells, suggesting that the STAT3/Jak2 pathway was not influenced by CK1. However, both AKT and p-AKT protein levels were regulated by CK1 expression, suggesting that the AKT pathway was involved in the CK1mediated glioma cell proliferation and metastasis. These results are consistent with reports from Manni et al. which demonstrated that CK1 $\alpha$ regulated the AKT pathway by activating the mechanistic target of rapamycin (mTOR) (21).

MMP2 has been shown to enhance the invasion of glioma cells by degrading the ECM (22). In the current study, MMP2 protein levels were regulated by CK1 expression. Suppression of AKT by the inhibitor API-1 reduced the levels of MMP2 expression, suggesting that MMP2 was regulated by CK1 through the AKT pathway, thereby promoting the invasion of glioma cells. Moreover, the mir-93-5p was reported to be involved in inhibition of proliferation and metastasis for glioma by targeting MMP2. Therefore, one possibility is that CK1 regulate the MMP2 expression by affect the mir-93-5p expression (23).

Glioblastoma multiforme (GBM) is the most aggressive type of brain tumor in adults and its extracranial seeding is rare. However, there has been a case report of glioblastoma in a 41-year-old man who experienced surgical resection and died 2 months after the diagnosis of spinal seeding (24). In addition, a boy with GBM developed increased abdominal distention and was clinically diagnosed with peritoneal metastases (25). Moreover, circulating tumor cells were identified in peripheral blood from 29 of 141 (20.6\%) GBM patients (26). All these reports demonstrated that glioma is not limited to the brain and can spread to extracranial organs. Indeed, the metastasis model using nude mice in our current study demonstrated that CK1 overexpression promoted glioma cell metastasis to the small intestine.

AKT regulates cell proliferation, growth, survival, and metabolism. The phosphorylation of Thr-308 in
AKT is used as a marker for AKT activation (27) and its phosphorylation is a prerequisite for kinase activation. In fact, AKT-dependent responses can be activated in the absence of ser 473 phosphorylation (28). However, the mechanisms by which CK1 enhances the phosphorylation of Thr-308 in AKT remain to be elucidate. Furthermore, the role of phosphoinositide dependent protein kinase 1 (PDK1) in the phosphorylation of Thr-308 in AKT is unclear and further investigations are warranted. This study demonstrated that CK1 promoted glioma proliferation and metastasis via the AKT-MMP2 pathway. Interestingly, Liang et al. showed that this pathway is also involved in vasculogenic mimicry (29).

The findings in this investigation demonstrated that CK1 plays an important role in cancer occurrence, development, and invasion of glioma cells. Additionally, the AKT-MMP2 pathway was shown to be closely related to CK1 expression in promoting the proliferation and metastasis of gliomas. Therefore, CK1 may be a novel therapeutic target for the treatment of gliomas. Further research is warranted to elucidate the mechanisms by which CK1 induces the occurrence, development, and metastasis of gliomas. Furthermore, many circRNA and lncRNA were reported to be involved in the proliferation and metastasis of glioma such as circCPA4 and LncRNA LINC00689. These RNAs could act as oncogenes or tumor suppressors in glioma progression (30-32). However, what connection between the CK1 and the RNAs still need further research.

\section{Acknowledgments}

Funding: None.

\section{Footnote}

Reporting Checklist: The authors have completed the ARRIVE reporting checklist. Available at http://dx.doi. org/10.21037/atm-21-935

Data Sharing Statement: Available at http://dx.doi. org/10.21037/atm-21-935

Conflicts of Interest: All authors have completed the ICMJE uniform disclosure form (available at http://dx.doi. org/10.21037/atm-21-935). The authors have no conflicts of interest to declare.

Ethical Statement: The authors are accountable for all 


\section{Page 12 of 13}

aspects of the work in ensuring that questions related to the accuracy or integrity of any part of the work are appropriately investigated and resolved. This study was approved by the research ethics committee of the Affiliated Hospital of Nantong University and written informed consent was obtained from each patient. All procedures were conducted in accordance with the Declaration of Helsinki (as revised in 2013). Animal care and experimental protocols were conducted following the guidelines established by the Shanghai Medical Experimental Animal Care Commission and study involving animals was approved by the Ethics Committee of Huashan Hospital affiliated to Fudan University (No. 2016-111).

Open Access Statement: This is an Open Access article distributed in accordance with the Creative Commons Attribution-NonCommercial-NoDerivs 4.0 International License (CC BY-NC-ND 4.0), which permits the noncommercial replication and distribution of the article with the strict proviso that no changes or edits are made and the original work is properly cited (including links to both the formal publication through the relevant DOI and the license). See: https://creativecommons.org/licenses/by-nc-nd/4.0/.

\section{References}

1. Ahn BY, Saldanha-Gama RF, Rahn JJ, et al. Glioma invasion mediated by the 775 neurotrophin receptor (p75(NTR)/CD271) requires regulated interaction with PDLIM1. Oncogene 2016;35:1411-22.

2. Paw I, Carpenter RC, Watabe K, et al. Mechanisms regulating glioma invasion. Cancer Lett 2015;362:1-7.

3. Knippschild U, Gocht A, Wolff S, et al. The casein kinase 1 family:participation in multiple cellular processes in eukaryotes. Cell Signal 2005;17:675-89.

4. Schittek B, Sinnberg T. Biological functions of casein kinase 1 isoforms and putative roles in tumorigenesis. Mol Cancer 2014;13:231.

5. Knippschild U, Wolff S, Giamas G, et al. The role of the casein kinase 1 (CK1) family in different signaling pathways linked to cancer development. Onkologie 2005;28:508-14.

6. Mack SC, Witt H, Piro RM, et al. Epigenomic alterations define lethal CIMP-positive ependymomas of infancy. Nature 2014;506:445-50.

7. Ciruelos Gil EM. Targeting the PI3K/AKT/mTOR pathway in estrogen receptor-positive breast cancer. Cancer Treat Rev 2014;40:862-71.

\section{Gao et al. CK1 promotes the proliferation and metastasis of glioma}

8. Sasaki T, Kuniyasu H. Significance of AKT in gastric cancer (Review). Int J Oncol 2014;45:2187-92.

9. Fresno Vara JA, Casado E, De Castro J, et al. PI3K/ Akt signalling pathway and cancer. Cancer Treat Rev 2004;30:193-204.

10. Ramachandran RK, Sorensen MD, Aaberg-Jessen $\mathrm{C}$, et al. Expression and prognostic impact of matrix metalloproteinase-2 (MMP-2) in astrocytomas. PLoS One 2017;12:e0172234.

11. Groblewska M, Siewko M, Mroczko B, et al. The role of matrix metalloproteinases (MMPs) and their inhibitors (TIMPs) in the development of esophageal cancer. Folia Histochem Cytobiol 2012;50:12-9.

12. Zhang $M$, Zhang $X$. Association of MMP-2 expression and prognosis in osteosarcoma patients. Int J Clin Exp Pathol 2015;8:14965-70.

13. Zhu Y, Yan L, Zhu W, et al. MMP2/3 promote the growth and migration of laryngeal squamous cell carcinoma via PI3K/Akt-NF-кB-mediated epithelial -mesenchymal transformation. J Cell Physiol 2019;1:1-9.

14. Zhou N, Yao Y, Ye H, et al. Abscisic-acid-induced cellular apoptosis and differentiation in glioma via the retinoid acid signaling pathway. Int J Cancer 2016;138:1947-58.

15. Sancho-Martinez I, Nivet E, Xia Y, et al. Establishment of human iPSC-based models for the study and targeting of glioma initiating cells. Nat Commun 2016;7:10743.

16. Fulcher LJ, Sapkota GP. Functions and regulation of the serine/threonine protein kinase CK1 family: moving beyond promiscuity. Biochem J 2020;477: 4603-21.

17. Hu Y, Song W, Cirstea D, et al. CSNK1alpha1 mediates malignant plasma cell survival. Leukemia 2015;29:474-82.

18. Varghese RT, Young S, Pham L, et al. Casein Kinase 1 Epsilon Regulates Glioblastoma Cell Survival. Sci Rep 2018;8:13621.

19. Huo XY, Zhang XY, Yuan F, et al. HOXB7 promotes proliferation and metastasis of glioma by regulating the Wnt/ $\beta$-catenin pathway. Eur Rev Med Pharmacol Sci 2019;23:2476-85.

20. Fan Z, Xu Q, Wang C, et al. A tropomyosin-like Meretrix meretrix Linnaeus polypeptide inhibits the proliferation and metastasis of glioma cells via microtubule polymerization and FAK/Akt/MMPs signaling. Int J Biol Macromol 2020;145:154-64.

21. Manni S, Carrino M, Manzoni M, et al. Inactivation of CK1alpha in multiple myeloma empowers drug cytotoxicity by affecting AKT and beta-catenin survival signaling pathways. Oncotarget 2017;8:14604-19.

22. Zhang JF, Wang P, Yan YJ, et al. IL33 enhances glioma 
cell migration and invasion by upregulation of MMP2 and MMP9 via the ST2-NF-kappaB pathway. Oncol Rep 2017;38:2033-42.

23. Wu H, Liu L, Zhu JM. MiR-93-5p inhibited proliferation and metastasis of glioma cells by targeting MMP2. Eur Rev Med Pharmacol Sci 2019;23:9517-24.

24. Kuo LT, Tsai SY, Yang CY, et al. Meningeal seeding from glioblastoma multiforme treated with radiotherapy and temozolomide. Asian J Surg 2017;40:61-5.

25. Narayan A, Jallo G, Huisman TA. Extracranial, peritoneal seeding of primary malignant brain tumors through ventriculo-peritoneal shunts in children:Case report and review of the literature. Neuroradiol J 2015;28:536-9.

26. Müller C, Holtschmidt J, Auer M, et al. Hematogenous dissemination of glioblastoma multiforme. Sci Transl Med 2014;6:247ra101.

27. Yoshioka Y, Suzuki T, Matsuo Y, et al. SMYD3-mediated lysine methylation in the $\mathrm{PH}$ domain is critical for activation of AKT1. Oncotarget 2016;7:75023-37.

Cite this article as: Gao HS, Lin SY, Han X, Xu HZ, Gao YL, Qin ZY. Casein kinase 1 (CK1) promotes the proliferation and metastasis of glioma cells via the phosphatidylinositol 3 kinasematrix metalloproteinase 2 (AKT-MMP2) pathway. Ann Transl Med 2021;9(8):659. doi: 10.21037/atm-21-935
28. Cailliau K, Lescuyer A, Burnol AF, et al. RasGAP Shields Akt from Deactivating Phosphatases in Fibroblast Growth Factor Signaling but Loses This Ability Once Cleaved by Caspase-3. J Biol Chem 2015;290:19653-65.

29. Liang X, Sun R, Zhao X, et al. Rictor regulates the vasculogenic mimicry of melanoma via the AKT-MMP-2/9 pathway. J Cell Mol Med 2017;21:3579-91.

30. Peng H, Qin C, Zhang C, et al. circCPA4 acts as a prognostic factor and regulates the proliferation and metastasis of glioma. J Cell Mol Med 2019;23:6658-65.

31. Liu X, Zhu Q, Guo Y, et al. LncRNA LINC00689 promotes the growth, metastasis and glycolysis of glioma cells by targeting miR-338-3p/PKM2 axis. Biomed Pharmacother 2019;117:109069.

32. Liu Q, Yu W, Zhu S, et al. Long noncoding RNA GAS5 regulates the proliferation, migration, and invasion of glioma cells by negatively regulating miR-18a-5p. J Cell Physiol 2018;234:757-68.

(English Language Editor: J. Toeh) 